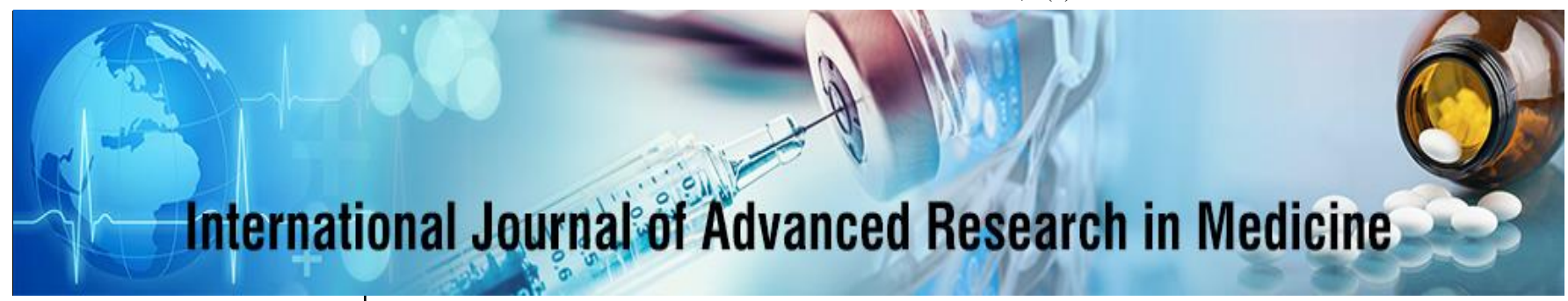

E-ISSN: 2706-9575

P-ISSN: 2706-9567

IJARM 2021; 3(1): 331-335

Received: 10-07-2020

Accepted: 15-09-2020

Maureen Edwards

Tutor

Department of Microbiology Srinivas institute of medical sciences \& Research Centre Mukka, Karnataka, India

Corresponding Author: Maureen Edwards

Tutor

Department of Microbiology Srinivas institute of medical sciences \& Research Centre Mukka, Karnataka, India

\section{Pattern in Minimum Inhibitory Concentration of Vancomycin and Tigecycline on Clinical Isolates of Enterococcus Species Isolated from Clinical Samples at our Tertiary Care Hospital}

\section{Maureen Edwards}

DOI: $\underline{\text { https://doi.org/10.22271/27069567.2021.v3.i1f.160 }}$

\begin{abstract}
Background: Genus Enterococcus have emerged as nosocomial pathogens. Urinary tract infections followed by intra-abdominal abscesses, Wounds, usually intra-abdominal or pelvic, are the next most common sites. Bacteremia is the third most common infection caused by Enterococci. Enterococcal meningitis is rare and is seen primarily in neonates and inpatients who have undergone complicated neurosurgical procedures ${ }^{[4]}$. There is an increasing role of Enterococci in nosocomial infections in the recent years mostly because of their intrinsic resistance to many clinically useful antibacterial drugs. One of the leading cause of nosocomial infection is Vancomycin-resistant Enterococci (VRE). For good therapy an effective, accurate and early detection of VRE along with Minimum Inhibitory Concentrations (MIC) is necessary. Tigecycline has a broad spectrum of activity against enterococci (vancomycin-susceptible). Haemolysin is a cytolytic protein and the producing strains of Enterococci have been shown to be associated within creased severity of infection ${ }^{[9]}$. In order to know the current scenario of minimum inhibitory concentration of Vancomycin and Tigecycline and the production of Haemolysin by some strains of Enterococci, the present study has been done.

Methods: This study was performed on Clinical isolates of Enterococcus which were received from tertiary care hospitals and processed at Department of Microbiology, Kasturba Medical College, Mangalore and Microbiology diagnostic laboratory (KMC hospital, Ambedkar circle), Mangalore. The study was done for a period of 6 months from Nov 2017-May2018. Convenient non-random sampling method was followed to collect clinical isolates. All isolates of Enterococcus from various clinical specimens like pus, deep tissue, urine, blood and body fluids were included in the study.

Result: Out of 100 Enterococcus isolates collected, majority were of E. faecium. Among all the isolates, $5 \%$ were resistant to vancomycin, but $100 \%$ sensitivity to tigecycline. Hemolysin produced by $46 \%$ of the total isolates. It has shown its association of the Enterococcus species to various antibiotics and their resistant pattern.
\end{abstract}

Keywords: MIC, Enterococcus, VRE

\section{Introduction}

Genus Enterococcus is Gram positive, ovoid shaped cocci which are arranged in pairs or short chains. ${ }^{[1]}$ Though they are the normal flora of the intestinal tract, oral cavity and vagina, but have emerged as nosocomial pathogens ${ }^{[2]}$. This genus is composed of 38 species, the most important of which are Enterococcus faecalis and Enterococcus faecium - both human intestinal colonizers ${ }^{[3]}$. Urinary tract infections followed by intra-abdominal abscesses are by far the most common enterococcal infections in humans majority being nosocomial. Wounds, usually intra-abdominal or pelvic, are the next most common sites. Bacteremia is the third most common infection caused by Enterococci. Enterococcal meningitis is rare and is seen primarily in neonates and in patients who have undergone complicated neurosurgical procedures ${ }^{[4]}$.

E. faecalis is isolated from approximately $80-90 \%$ of human infections and E. faecium from most of the rest. Enterococcus is mainly isolated from urine, pus, blood and body fluids. ${ }^{[5]}$ There is an increasing role of Enterococci in nosocomial infections in the recent years mostly because of their intrinsic resistance to many clinically useful antibacterial drugs including 
$\beta$-lactam antibiotics, aminoglycosides and glycopeptides like vancomycin. The unyielding response to the treatment owe to the high mortality rate of Enterococcal infections ${ }^{[5]}$. Tigecycline is a new, semi synthetic glycylcycline ${ }^{[6]}$. Resistance to the tetracycline class is mediated by ribosomal protection mechanism or by efflux. Tigecycline has more potent activity against tetracycline-resistant organisms. Based on in vitro susceptibility data, tigecycline has a broad spectrum of activity against enterococci (vancomycinsusceptible) [7]. Quinupristin/dalfopristin, linezolid, daptomycin, tigecycline and vancomycin are FDA approved agents as appropriate choices for treatment of Gram-positive pathogens ${ }^{[7]}$.

The third leading cause of nosocomial infection is Vancomycin- resistant Enterococci (VRE). Occurrence of VRE is a persisting clinical problem in all geographic areas and continues to be exacerbated by clonal dissemination within the health care facility leading to limited therapeutic options. Steady pandemic spread of VRE along with acquisition of resistance to newer antimicrobials warrants continued surveillance of these versatile pathogens. For good therapy an effective, accurate and early detection of VRE along with Minimum Inhibitory Concentrations (MIC) is necessary ${ }^{[8]}$.

Haemolysin is a cytolytic protein capable of lysing human, horse and rabbit erythrocytes ${ }^{[9]}$. Haemolysin producing strains of Enterococci have been shown to be virulent in animal models and human infections and to be associated with increased severity of infection ${ }^{[9]}$.

In order to know the current scenario of minimum inhibitory concentration of Vancomycin and Tigecycline and the production of Haemolysin by some strains of Enterococci, the present study is undertaken to determine the Minimum Inhibitory Concentration (MIC) of Tigecycline and Vancomycin on clinical isolates of Enterococcus and to correlate between hemolysin production and drug resistance pattern.

\section{Materials and Methods}

This study was performed on Clinical isolates of Enterococcus which were received from tertiary care hospitals and processed at Department of Microbiology, Kasturba Medical College, Mangalore and Microbiology diagnostic laboratory (KMC hospital, Ambedkar circle), Mangalore. The study was done for a period of 6 months from Nov 2017-May 2018.

Convenient non-random sampling method was followed to collect clinical isolates. All isolates of Enterococcus from various clinical specimens like pus, deep tissue, urine, blood and body fluids were included in the study. All the media and chemicals used in the study were procured from HI Media Laboratories Pvt Ltd. Mumbai, India. Urine samples were cultured on UTI chrome agar and Cysteine Lactose Electrolyte Deficient medium by semi-quantitative method. Direct microscopic examination of the urine samples was also done. Blood samples were processed in automated systems (BacT/ALERT3D). They were then inoculated on Blood agar and MacConkey's agar. All the other samples were inoculated on Blood agar and MacConkey's agar. The plates were incubated at $37^{\circ} \mathrm{C}$ for 24 hours and growth was examined. Identification of genus Enterococcus was done by colony morphology, Gram's staining, Bile Esculin test and speciation was done by Arabinose fermentation test.
Enterococcus isolates were subjected to antibiotic susceptibility testing by modified Kirby-Bauer disc diffusion method, and results were interpreted according to Clinical Laboratory Standards Institute (CLSI) guidelines.

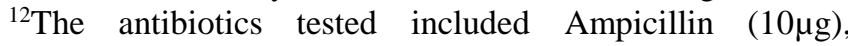

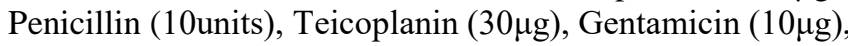
Tetracycline $(30 \mu \mathrm{g})$, erythromycin $(15 \mu \mathrm{g})$, Chloramphenicol $(30 \mu \mathrm{g})$, Ciprofloxacin $(5 \mu \mathrm{g})$, Vancomycin $(30 \mu \mathrm{g})$, Nitrofurantoin $(300 \mu \mathrm{g})$ and Streptomycin. All antibiotic susceptibility tests were conducted using ATCC E. faecalis 29212 as control. The antibiotics were purchased from Hi Media Laboratories, Mumbai, India.

Dilution susceptibility testing methods are used to determine the minimal concentration, usually in micro gram per milliliter, of an anti-microbial agent required to inhibit or kill a microorganism.

In the agar dilution method for minimum inhibitory concentrations, a standardized suspension of bacteria was inoculated onto a series of Mueller-Hinton agar plates, containing different concentrations (two fold serial dilutions) of Vancomycin and Tigecycline. Enterococcus isolates were inoculated in Mueller-Hinton broth and incubated for 4 hours at $37^{\circ} \mathrm{C}$ and adjusted to $0.5 \mathrm{McFarland}$ Standard which were then inoculated onto the MHA plates. MIC control strain ATCC E. feacalis 29212 and ATCC E. feacalis 51299 was used. MIC of both Vancomycin and Tigecycline was interpreted for all VRE isolates by agar dilution method according to the guidelines established by the CLSI ${ }^{25]}$.

MIC of Vancomycin and Tigecycline was determined by $\mathrm{E}$ test for all Enterococci isolates. A lawn culture of Enterococci, 0.5 McFarland's standard was made on 5\% Mueller Hinton agar. The E strip obtained from HI Media were placed on the inoculated plates. The plates were then incubated at $37^{\circ} \mathrm{C}$ in $5 \% \mathrm{CO}_{2}$ for 24 hours. The MIC was read where the elliptical zone intersected the MIC scale on the E-strip. Vancomycin sensitive strain of E. feacalis ATCC 29212 was used as a negative control and E. feacalis ATCC 51299, vancomycin resistant strain was used as a positive control (CLSI2017). The results for Vancomycin were interpreted as sensitive (MIC<4), intermediate (MIC 816) and resistant (MIC>32) based on CLSI guidelines (2017) and for Tigecycline $\mathrm{MIC} \leq 0.5$ was considered as sensitive.

Hemolysin production was detected by inoculating enterococci samples onto freshly prepared Mueller-Hinton blood agar supplemented with 5\% human blood. Plates were incubated overnight at $37^{\circ} \mathrm{C}$ in a carbon dioxide chamber and evaluated at 24 and 48 hours. A clear zone of $\beta$ hemolysis around the streak on human blood agar was considered to be a positive indication of hemolysin production.

\section{Results}

A total of 100 Enterococcus isolates were collected, among which $46 \%$ were of E. faecalis, and $54 \%$ were of E. faecium out of which 41 strains were isolated from males and 59 strains from females, as shown in figure 1. All the strains used in this study were isolated from different clinical specimens (Table 1). Figure 2 shows the distribution of Enterococci based on gender. 
Table 1: Enterococci isolated from various clinical specimens.

\begin{tabular}{|c|c|}
\hline Specimens & Enterococci (\%) \\
\hline Urine, Kidney stone & 51 \\
\hline Deep Tissue & 6 \\
\hline Wound swab & 11 \\
\hline HVS, Umbilical swab & 8 \\
\hline Bronchoalveolar Lavage & 1 \\
\hline Pus, peritoneal fluid & 14 \\
\hline Blood, Suction tip, bile & 4 \\
\hline ET aspirate & 5 \\
\hline
\end{tabular}

Polymorph nuclear leucocytes (PMNL) was found to be associated with 40 of the urinary isolates, which comprised 20 E. faecalis and 20 of E. faecium. Among the seven high vaginal swabs that showed growth of Enterococcus spp. 5 had PMNL. These five isolates included 3 of E. faecalis and 2 of E. faecium.

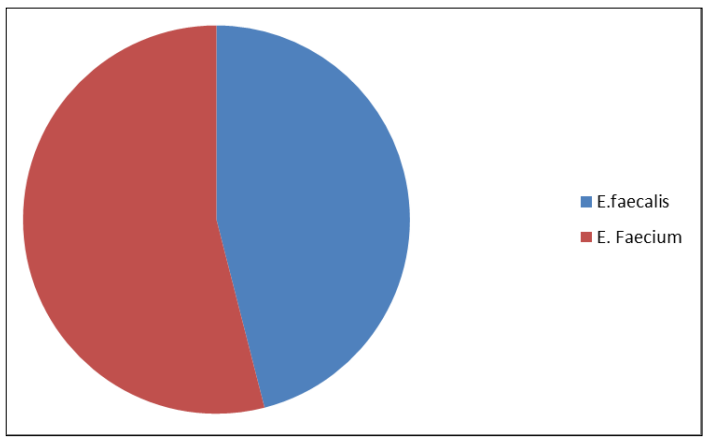

Fig 1: Enterococci

$1 \%$ of E. faecalis and $2 \%$ of E. faecium strains were resistant to vancomycin by disc diffusion method, and these were isolated from pus and deep tissue. MIC values of two

isolates showed greater $\mathrm{MIC} \geq 32 \mu \mathrm{g} / \mathrm{ml}$ and one with MIC $\geq 8 \mu \mathrm{g} / \mathrm{ml}$.

Antimicrobial susceptibility pattern for the Enterococcus isolates collected shown in Table 2.Of 100 isolates,

Table 2: Antimicrobial susceptibility pattern for Enterococcus.

\begin{tabular}{|c|c|c|}
\hline \multirow{2}{*}{ Antibiotics tested } & \multicolumn{2}{|c|}{ Percentage } \\
\cline { 2 - 3 } & Sensitive & Resistant \\
\hline Amikacin & 31 & 69 \\
\hline Amoxyclav & 55 & 45 \\
\hline Ampicillin & 51 & 49 \\
\hline Erythromycin & 30 & 70 \\
\hline High-level Gentamicin & 55 & 45 \\
\hline High-level Streptomycin & 44 & 56 \\
\hline Imipenem & 58 & 42 \\
\hline Meropenem & 50 & 50 \\
\hline Nitrofurantoin & 75 & 25 \\
\hline Piperacillin & 35 & 65 \\
\hline Piperacillin-tazobactam & 60 & 40 \\
\hline Teicoplanin & 90 & 10 \\
\hline Vancomycin & 95 & 5 \\
\hline
\end{tabular}

Out of 100 isolates, for amikacin, amoxyclav and nitrofurantoin sensitivity was $31 \%, 55 \%$, and $75 \%$, respectively. $60 \%$ of these isolates were sensitive to Piperacillin-tazobactam, but $40 \%$ showed resistance. $58 \%$ were sensitive to imipenem and meropenem was sensitive to $50 \%$ Enterococcus isolates. 5\% of the total isolates showed Vancomycin resistance. $44 \%$ and $55 \%$ of the isolates showed resistance to High-level streptomycin and Highlevel gentamicin, respectively. Intermediate susceptibility was seen for antibiotics like amikacin (2\%), meropenem (2\%), piperacillin-tazobactam (2\%), High-level gentamicin $(2 \%)$, erythromycin $(2 \%)$, piperacillin $(5 \%)$ and teicoplanin $(2 \%)$.

\begin{tabular}{|c|c|c|c|}
\hline Clinical conditions & No. of samples & Risk factors & Recovery \\
\hline Acute coronary syndrome & 1 & Sepsis pneumonia Type 2 RR & No (expired) \\
\hline Meningitis & 1 & TB & Yes \\
\hline Hydroureteronephrosis & 1 & Ureteric calculus & Yes \\
\hline Urinary tract infection & 27 & Chronic cervicitis, DM, HTN, primigravida cervical incompetence ARR & Yes \\
\hline Cholangitis & 1 & DM, IHD, BPH & Yes \\
\hline Abscess & 1 & Perianal abscess IHD & Yes \\
\hline Ulcer & 2 & Sepsis Type 2 DM DKA PVD & Yes \\
\hline Pulmonary infection & 6 & BPH Type 2 DM HTN DI Hypertension & Yes \\
\hline
\end{tabular}

MIC of Vancomycin and tigecycline was done by E-strip, and the percentage susceptibility of Enterococcus isolates is shown in Table 3. All the strains were within the range and susceptible to tigecycline, whereas, three isolates showed the higher threshold for Vancomycin with two of them MIC of $32 \mu \mathrm{g} / \mathrm{ml}$ and one with a MIC of $8 \mu \mathrm{g} / \mathrm{ml}$.
Table 3: Minimum inhibitory concentrations of vancomycin and tigecycline against Enterococcus by E-test

\begin{tabular}{|c|c|c|}
\hline \multirow{2}{*}{ No. of dilutions } & \% of isolates susceptible at the MIC $(\boldsymbol{\mu g} \mathbf{m} \mathbf{m})$ \\
\cline { 2 - 3 } & $\begin{array}{c}\text { Vancomycin } \\
\text { Range (1-4) }\end{array}$ & $\begin{array}{c}\text { Tigecycline } \\
\text { Range }(\leq \mathbf{0 . 2 5})\end{array}$ \\
\hline 0.032 & 0 & 30 \\
\hline 0.064 & 0 & 25 \\
\hline 0.125 & 0 & 5 \\
\hline 0.25 & 0 & 40 \\
\hline 0.50 & 0 & 0 \\
\hline 0.75 & 10 & 0 \\
\hline 1 & 30 & 0 \\
\hline 1.5 & 37 & 0 \\
\hline 2 & 7 & 0 \\
\hline 4 & 12 & 0 \\
\hline 8 & 1 & 0 \\
\hline 16 & 1 & 0 \\
\hline 32 & 2 & 0 \\
\hline
\end{tabular}


Table 4 shows MIC of linezolid and tigecycline against MRSA done by agar dilution. All the isolates were within the susceptible MIC range for linezolid. For tigecycline, three strains showed MIC $>0.5 \mu \mathrm{g} / \mathrm{ml}$.

Table 4: Minimum inhibitory concentrations of Vancomycin and Tigecycline against Enterococcus by agar dilution method

\begin{tabular}{|c|c|c|}
\hline \multirow{2}{*}{ No. of dilutions } & \multicolumn{2}{|c|}{$\begin{array}{c}\text { \% of isolates susceptible at the MIC } \\
(\boldsymbol{\mu g} / \mathbf{m l})\end{array}$} \\
\cline { 2 - 3 } & $\begin{array}{c}\text { Vancomycin } \\
\text { Range (1-4) }\end{array}$ & $\begin{array}{c}\text { Tigecycline } \\
\text { Range ( } \leq \mathbf{0 . 2 5})\end{array}$ \\
\hline 0.032 & 0 & 5 \\
\hline 0.064 & 0 & 5 \\
\hline 0.125 & 0 & 14 \\
\hline 0.25 & 0 & 76 \\
\hline 0.50 & 12 & 0 \\
\hline 1 & 30 & 0 \\
\hline 2 & 14 & 0 \\
\hline 4 & 40 & 0 \\
\hline 8 & 1 & 0 \\
\hline 16 & 1 & 0 \\
\hline 32 & 2 & 0 \\
\hline
\end{tabular}

Haemolysin production among 46\% isolates. Hemolytic activity was seen in both the species of Enterococcus. $28 \%$ of E. faecalis strains and $19 \%$ OF E. faecium produced the virulence factor.

\section{Discussion}

Enterococcus is one of the major pathogens affecting all age groups. E. faecium is more resistant than E. faecalis. The isolation rate of $E$. faecalis was more than that of $E$. faecium. However, studies carried out in North India have shown E. faecium to be responsible for a large number of infections than E. faecalis. In our study, $46 \%$ of the total isolates were tested positive for haemolysin. It was also observed that nearly $28 \%$ of E. faecalis isolates possessed hemolysin, whereas this virulence factor was lesser in case of E. faecium isolates. This may be one of the reasons why the species of $E$. faecalis is responsible for a large number of infections. The ability of haemolysin production helps the organisms to acquire nutrition in the host tissues and for the further spread of infection in the host body, thus increasing the infection severity.

Antibiotic resistance among Enterococci is a global problem. In our study, the highest resistance was seen against Erythromycin, which is in agreement with other studies carried out in India. In the current study, 45\% and $56 \%$ of the total Enterococcus species were resistant to high-level gentamycin and high-level streptomycin respectively.

Agar dilution done for Vancomycin and tigecycline showed MIC values within the susceptible range, except $5 \%$ isolates which showed resistance to Vancomycin suggesting they belong to Vancomycin-resistant Enterococcus (VRE). VRE is expected to be a significant problem in the coming years, and hence it is essential that proper measures have to be taken in all healthcare settings to contain the dissemination of the resistant strains. For all the enterococcal isolates, routine testing should be done at least by Vancomycin agar screen test; Vancomycin drug should be used judiciously. Rapid detection and treatment of VRE infected patients will help in limiting the spread of VRE. Studies are necessary to characterize the virulence factors and the drug-resistance genes of enterococcal isolates by the known molecular methods to understand precisely their role in the pathogenesis of nosocomial infections.

\section{Conclusion}

The present study shows the susceptibility pattern trend for vancomycin and tigecycline. It was found that among all the isolates, $5 \%$ were resistant to vancomycin, but $100 \%$ sensitivity to tigecycline. This conclusion was derived after susceptibility testing was done by both agar dilution and Etest.

Hemolysin production confirmed by a phenotypic method on human blood agar. The results were found to be similar to other studies. This virulence factor was produced by $46 \%$ of the total isolates. This test shows the association of the Enterococcus species with various antibiotics and their resistant pattern.

Vancomycin-Resistant Enterococci (VRE) were also isolated during this study. This can be further confirmed by genotypic methods.

The study will help to determine the present trend of MIC values of Vancomycin and Tigecycline for the clinical isolates of Enterococcus species from our tertiary care hospitals.

\section{References}

1. Winn WC Jr, Allen SD, Jande WH, Koneman EW, Schreckenberger PC. The gram positive cocci Part II: Streptococci, Enterococci and the Streptococcus like bacteria. Koneman's Color Atlas and Text book of Diagnostic Microbiology 6th ed. Lippincott, Philadelphia 2006;672-764.

2. Kheya Mukherjee, Debojyoti Bhattacharjee, Goutam Chakraborti, Shiv sekhar Chatterjee. Prevalence and antibiotic susceptibility pattern of Enterococcus species from various clinical samples in a tertiary care hospital in Kolkata. International Journal of Contemporary Medical Research 2016;3(6):1565-1567.

3. $\mathrm{Vu} \mathrm{J}$, and Carvalho J. Enterococcus : review of its physiology, pathogenesis, diseases and the challenges it poses for clinical microbiology 2011;6(5):357-366.

4. Moellering RC. State-of-The-Art Clinical Emergence of Enterococcus as a Significant Pathogen 2014;14(6): 1173-1176.

5. Shah L, Mulla S, Patel KG, Rewadiwala S. Prevalence of Enterococci with higher resistance level in a tertiary care hospital: a matter of concern. Natl J Med Res. 2012;2:25-27.

6. Iseri Latife, Sahin Esra, DolapciIstar, Yuruken Zehra. Minimum inhibitory concentration values and problematic disk breakpoints of tigecycline against vancomycin and/or high-level aminoglycoside-resistant enterococci. Alexandria Journal of Medicine 2016;52: 125-129.

7. Lentino JR, Narita M, Yu LY. New Antimicrobial Agents as Therapy For Resistant Gram-Positive Cocci. Eur J Clin Microbiol Infect Dis 2008;27(1):3-15.

8. Tripathi A, Shukla SK, Singh A, Prasad KN. A new approach of real-time polymerase chain reaction in detection of vancomycin-resistant Enterococci and its comparison with other Methods. Indian $\mathbf{J}$ Med Microbiol 2013;31:47-52.

9. Giridhara Upadhyaya PM, Ravikumar KL, Umapathy B L. Review of virulence factors of enterococcus: An emerging nosocomial pathogen. Indian J Med Micro 
boil 2009;27:301-5.

10. Po-Ren Hsueh, Wen-Huei Chen, Lee-Jene Teng, KwenTay Luh. Nosocomial infections due to methicillinresistant Staphylococcus aureus and Vancomycinresistant enterococci at a university hospital in Taiwan from 1991-2003: resistance trends, antibiotic usage and in-vitro activities of newer anti-microbial agents IJAA 2005;26:43-49.

11. Kheya Mukherjee, Debojyoti Bhattacharjee, Goutam Chakraborti, Shiv sekhar Chatterjee. Prevalence and antibiotic susceptibility pattern of Enterococcus species from various clinical samples in a tertiary care hospital in Kolkata. International Journal of Contemporary Medical Research 2016;3(6):1565-1567.

12. Sofia Maraki, George Samonis, Dimitra Dimopoulou, and Elpis Mantadakis Susceptibility of GlycopeptideResistant Enterococci to Linezolid, Quinupristin/dalfopristin, Tigecycline and Daptomycin in a Tertiary Greek Hospital Infect Chemotherapy 2014;46(4):253-256.

13. Joseph R. Lentino, M.D., Ph.D., Masashi Narita, M.D., and Victor L.Yu, M.D. New antimicrobial agents as therapy for resistant Gram-positive Cocci 2000.

14. CLSI. Performance Standards for Antimicrobial Susceptibility Testing; Twenty-seventh Informational Supplement. CLSI document M100-S27. Wayne, PA: Clinical and Laboratory Standards Institute 2017.

15. Vergis EN, Nathan S, Joseph WC, Hayden MK, Syndman DR, Zervos et al. Association between the presence of Enterococcal virulence factors gelatinase, haemolysin and enterococcal surface protein and mortality among patients with bacteremia due to Enterococcus faecalis. Clin Infect Dis 2002;35:570-5.

16. Alotaibi FE, Bukhari EE. Emergence of Vancomycinresistant Enterococci at a Teaching Hospital, Saudi Arabia. Chin Med J 2017;130:340-6.

17. Papadimitriou-Olivgeris M, Kolonitsiou F, Zerva L, Lebessi E, Koutsia C, Drougka E et al. Activity of vancomycin, linezolid, and daptomycin against staphylococci and enterococci isolated in 5 Greek hospitals during a 5-year period (2008-2012). Diagnostic Microbiology and Infectious Disease. 2015;83(4):386-388.

18. Klare I, Fleige C, Geringer U, Witte W, Werner G. Performance of three chromogenic VRE screening agars, two Etest ${ }^{\circledR}$ vancomycin protocols, and different microdilution methods in detecting vanB genotype Enterococcus faecium with varying vancomycin MICs. Diagnostic Microbiology and Infectious Disease 2012;74(2):171-176.

19. Denys G, Koch K, Dowzicky M. Distribution of resistant gram-positive organisms across the census regions of the United States and in vitro activity of tigecycline, a new glycylcycline antimicrobial. American Journal of Infection Control 2007;35(8):521526.

20. Bartash R, Nori P. Beta-lactam combination therapy for the treatment of Staphylococcus aureus and Enterococcus species bacteremia: A summary and appraisal of the evidence. International Journal of Infectious Diseases. 2017;1;63:7-12. Available from, DOI: 10.1016/j.ijid.2017.07.019
21. George A. Pankey; Tigecycline, Journal of Antimicrobial Chemotherapy, 2005;56(3-1);470-480.

22. Lentino J, Narita M, Yu V. New antimicrobial agents as therapy for resistant gram-positive cocci. European Journal of Clinical Microbiology \& Infectious Diseases. 2007;27(1):3-15.

23. Toru, M, Beyene G, Kassa T, Gizachew Z, Howe R and Yeshitila B. Prevalence and phenotypic characterization of Enterococcus species isolated from clinical samples of pediatric patients in Jimma University Specialized Hospital, south west Ethiopia. BMC Research Notes 2018;11(1). 